\title{
NECESSARY AND SUFFICIENT RESTRICTIONS FOR EXISTENCE OF A UNIQUE FOURTH MOMENT OF A UnIVARIATE GARCH $(\mathrm{P}, \mathrm{Q})$ PROCESS
}

\author{
PETER A. ZADROZNY \\ CESIFO WORKING PAPER NO. 1505 \\ CATEGORY 10: EMPIRICAL AND THEORETICAL METHODS \\ JULY 2005 \\ - from the CESifo website: www.CESifo.de
}




\title{
NECESSARY AND SUFFICIENT RESTRICTIONS FOR EXISTENCE OF A UNIQUE FOURTH MOMENT OF A UNIVARIATE GARCH $(\mathrm{P}, \mathrm{Q})$ PROCESS
}

\begin{abstract}
A univariate $\operatorname{GARCH}(\mathrm{p}, \mathrm{q})$ process is quickly transformed to a univariate autoregressive moving-average process in squares of an underlying variable. For positive integer $\mathrm{m}$, eigenvalue restrictions have been proposed as necessary and sufficient restrictions for existence of a unique mth moment of the output of a univariate GARCH process or, equivalently, the $2 \mathrm{mth}$ moment of the underlying variable. However, proofs in the literature that an eigenvalue restriction is necessary and sufficient for existence of unique 4th or higher even moments of the underlying variable, are either incorrect, incomplete, or unecessarily long. Thus, the paper contains a short and general proof that an eigenvalue restriction is necessary and sufficient for existence of a unique 4th moment of the underlying variable of a univariate GARCH process. The paper also derives an expression for computing the 4th moment in terms of the GARCH parameters, which immediately implies a necessary and sufficient inequality restriction for existence of the 4th moment. Because the inequality restriction is easily computed in a finite number of basic arithmetic operations on the GARCH parameters and does not require computing eigenvalues, it provides an easy means for computing "by hand" the 4th moment and for checking its existence for low-dimensional GARCH processes. Finally, the paper illustrates the computations with some $\operatorname{GARCH}(1,1)$ processes reported in the literature.
\end{abstract}

JEL Code: C32, C63, G12.

Keywords: state-space form, Lyapunov equations, nonnegative and irreducible matrices.

\author{
Peter A. Zadrozny \\ Bureau of Labor Statistics \\ 2 Massachusetts Ave., NE \\ Washington, DC 20212 \\ USA \\ zadrozny_p@bls.gov
}

The paper represents the author's views and does not necessarily represent any official positions of the Bureau of Labor Statistics. Thanks to Heinz Neudecker for helpful correspondence. 


\section{Introduction.}

A univariate $\operatorname{GARCH}(p, q)$ process (Engle, 1982; Bollerslev, 1986) is quickly transformed to a univariate autoregressive moving-average (ARMA) process in squares of an underlying variable. Henceforth, for brevity, unless otherwise qualified, we indicate "GARCH$(p, q)$ " by "GARCH." GARCH processes and their generalizations (Mittnik, Paolella, and Rachev, 2002) have been used to model volatilities or time-varying variances of underlying variables, usually financial-asset returns or residuals from estimated time-series models. GARCH processes are usually considered to have Gaussian or normally distributed disturbances, however, because asset returns can have large volatilities, such as the unexpected large drop in the U.S. stock market in october 1987, their distributions are often ascribed "heavier" tails than implied by the Gaussian distribution. Thus, Mandelbrot (1963a,b) studied asset-return distributions using stable-Paretian distributions. More recently, McCulloch (1997), Rachev and Mittnik (2000), and others studied time series of asset returns using GARCH and other processes driven by stable-Paretian and other heavy-tailed disturbances. Thus, it is useful to have a method for easily computing the 4th moment of an underlying variable of a univariate GARCH process, to check whether it exists and, if so, whether it indicates heavier than Gaussian tails. All the moments considered here are unconditional.

For positive integer $\mathrm{m}$, eigenvalue restrictions have been proposed as necessary and sufficient conditions for existence of a unique $2 \mathrm{mth}$ moment of the underlying variable of a univariate GARCH process. Proofs in the literature that an eigenvalue restriction is necessary and sufficient for existence of unique 4th or higher even moments of the underlying variable of a GARCH process are either incorrect, incomplete, or unecessarily long. Before detailing the present paper's contribution to this literature, we clarify our use of the term "4th moment." A GARCH process is a type of ARMA process which linearly transforms squared disturbances, $\varepsilon_{\mathrm{t}}^{2}$, to squared variables, $y_{t}^{2}$, so that the process nonlinearly transforms unsquared or underlying disturbances, $\varepsilon_{\mathrm{t}}$, to unsquared or underlying variables, $\mathrm{y}_{\mathrm{t}}$. Thus, the "2mth moment of the underlying variable of a GARCH process" is the $2 \mathrm{mth}$ moment of $y_{t}$ and is equivalent to the mth moment of $y_{t}^{2}$. In this regard, a GARCH process is (covariance) stationary if and only if the underlying variable, $\mathrm{y}_{\mathrm{t}}$, has a 2nd moment. Thus, unless otherwise qualified, "4th moment" means the 4 th moment of $y_{t}$. 
The paper contains a short and general proof that an eigenvalue restriction is necessary and sufficient for existence of the unique 4th moment of the underlying variable of a univariate GARCH process. The paper derives an expression for computing the 4th moment in terms of the GARCH parameters, which immediately implies a necessary and sufficient inequality restriction for checking the moment's existence. Because the eigenvalue and inequality restrictions are separately necessary and sufficient for the moment's existence, they are equivalent. Because the inequality restriction is easily computed in a finite number of basic arithmetic operations on the GARCH parameters and does not require computing eigenvalues, it provides an easy means for computing "by hand" the 4th moment and for checking its existence for low-dimensional GARCH processes.

The recent literature contains the following related results. In the following, all statements of "necessity" and "sufficiency" refer to the existence of the 4th moment of the underlying variable of a general univariate GARCH process.

He and Terasvirta (1999) stated an inequality restriction (theorem 1, p. 827) on a univariate GARCH process, which they claim (p. 833-840) is necessary and sufficient. Ling and McAleer (2002) question ( $p .724$ and $p$. 728, note 1 ) whether He and Terasvirta's necessity proof is complete. In any case, because they do not use a state-space representation, He and Terasvirta's discussion is unnecessarily long. Karanasos (1999) stated (theorem 3.1, p. 66) an inequality restriction and proved (pp. 73-74) its necessity, but, as Ling and McAleer noted (pp. 723-724), he did not prove its sufficiency. Ling (1999) stated and proved (theorem 6.2, p. 702) an eigenvalue restriction's sufficiency.

Ling and McAleer (2002) stated (theorem 2.1, pp. 723-724) an eigenvalue restriction and purportedly proved ( $p p .726-727$ ) its necessity. However, they actually proved necessity only for the special case $\alpha_{1}>0$. Their proof works if and only if (iff) $\alpha_{1}>\odot$ or $\beta_{1}>\odot$, but not if $\alpha_{1}=\beta_{1}=\odot$. Ling and McAleer claim ( $p$. 727) their proof still works if $\alpha_{1}=\beta_{1}=0$, but as written it does not because it requires a vector $R$ to have all positive elements after a certain number of repeated steps and this is not the case if $\alpha_{1}=\beta_{1}=0$. By contrast, the proof given here holds for any univariate GARCH process.

Eigenvalue restrictions require computing eigenvalues, which can generally be done analytically "by hand" only for matrices no larger than three dimensional, hence, for GARCH processes with no more than three lags. 
Thus, generally, eigenvalue restrictions can be checked only numerically on a computer. By contrast, as illustrated in section 4, the present necessary and sufficient inequality restriction for the 4th moment's existence is easily checked "by hand" in a finite number of basic arithmetic operations.

The remainder of the paper is organized as follows. Section 2 states a univariate GARCH process in state-space form in order to derive the 4thmoment inequality restriction. Section 3 proves that the inequality restriction is necessary and sufficient for existence of a unique 4th moment of the underlying variable of a univariate GARCH process of any degree. Section 3 also proves that the 4 th-moment inequality restriction is equivalent to an eigenvalue restriction. Section 4 numerically illustrates the 4th-moment inequality restriction with $\operatorname{six} \operatorname{GARCH}(1,1)$ processes from the literature. Section 5 contains concluding remarks.

\section{State-Space Form of a $\operatorname{GARCH}(p, q)$ Process.}

For discrete-time periods $t$, let $y_{t}^{2}=\varepsilon_{t}^{2} h_{t}$ denote the square of the underlying variable in the process, where $h_{t}$ is generated by the univariate GARCH process

$$
\mathrm{h}_{\mathrm{t}}=\alpha_{\Theta}+\sum_{\mathrm{i}=1}^{\mathrm{n}} \alpha_{\mathrm{i}} \varepsilon_{\mathrm{t}-\mathrm{i}}^{2} \mathrm{~h}_{\mathrm{t}-\mathrm{i}}+\sum_{\mathrm{i}=1}^{\mathrm{n}} \beta_{\mathrm{i}} \mathrm{h}_{\mathrm{t}-\mathrm{i}},
$$

$\alpha_{i}$ and $\beta_{i}$ are constant parameters, $n=\max (p, q), \alpha_{i}=0$ for $i>p$ if $p<n, \beta_{i}$ $=\odot$ for $i>q$ if $q<n$, and $\varepsilon_{t}$ is a disturbance. Although the aim is to verify necessary and sufficient restrictions on the $\operatorname{GARCH}(p, q)$ parameters for existence of the 4 th moment of the underlying variable, $E y_{t}^{4}=E\left(\varepsilon_{t}^{4} h_{t}^{2}\right)$, because we assume the disturbance's 4 th moment, $E \varepsilon_{t}^{4}$, exists, we concentrate on restrictions for existence of $E h_{t}^{2}$.

Notation is simplified but no generality is lost when process (2.1) is written as a $\operatorname{GARCH}(n, n)$ process. The proofs in section 3 do not depend on the presence of the second summation in (2.1), $\sum_{i=1}^{n} \beta_{i} h_{t-i}$, hence, on whether $q \geq$ 1 or $q \geq 0$. The following GARCH assumptions allow $q \geq 0$, but require $p \geq 1$.

We assume the following for $\operatorname{GARCH}$ process $(2.1)$ : (i) $n=\max (p, q)$, for $p \geq 1$ and $q \geq 0$; (ii) $\alpha_{\theta}>0$, (iii) $\alpha_{i} \geq 0$ and $\beta_{i} \geq 0$, for $i=1, \ldots, n$; (iv) $\alpha_{i}>\odot$, for one or more $i=1, \ldots, n$; (v) $\alpha_{n}>\odot$ or $\beta_{n}>\odot$, or both; and, (vi) 
$\varepsilon_{\mathrm{t}}$ is distributed identically, independently, with zero mean, $E \varepsilon_{\mathrm{t}}=0$, finite positive variance, $\sigma_{\varepsilon}^{2}=E \varepsilon_{\mathrm{t}}^{2}>0$, and finite positive 4th moment, $\sigma_{\varepsilon}^{4}=E \varepsilon_{\mathrm{t}}^{4}>$ $\odot$, which is the central and noncentral 4th moment of $\varepsilon_{t}$ because $\varepsilon_{t}=\odot$. As usual, without loss of generality, we set $\sigma_{\varepsilon}^{2}=1$ and, thus, effectively merge $\sigma_{\varepsilon}^{2}$ into $\alpha_{i}$.

Assumption ( $v$ ) is convenient but unnecessary, because if $\alpha_{n}=\beta_{n}=0$, then, assumption (iv) guarantees that we can reduce $n$ until $\alpha_{i}>\odot$ for some $i$ $=1, \ldots, \mathrm{n}-1$. We take " $\varepsilon_{\mathrm{t}}$ is distributed independently" to mean that $\varepsilon_{\mathrm{t}}$ is distributed independently not just of past values of itself but also of past values of $h_{t}$. We do not need any particular distributional assumption such as Gaussianity.

Finally, we assume GARCH process (2.1) is stationary. Throughout, by "stationarity" we mean weak or covariance stationarity. By constrast, for example, Nelson (1990) considers strong stationarity of $\operatorname{GARCH}(1,1)$ processes. Following Milhoj (1985), Bollerslev (1986) proved that, for $\alpha_{i} \geq 0$ and $\beta_{i} \geq 0$, process (2.1) is stationary iff (vii) $\sum_{i=1}^{n} f_{i}<1$, where $f_{i}=\alpha_{i}+\beta_{i}$. Although the final theorem 4 here proves that GARCH process (2.1) is stationary if $E h_{t}^{2}$ exists, we assume stationarity in order to simplify the discussion.

In sum, we assume (i) to (vii) for GARCH process (2.1) and call these "the GARCH assumptions."

If GARCH process (2.1) is stationary, then, its mean, $E h_{t}=\mu$, exists, is positive, and is given by

$$
\mu=\alpha_{0} /\left(1-\sum_{i=1}^{n} f_{i}\right)
$$

Let a tilde denote a mean-adjusted variable, so that $\tilde{h}_{t}=h_{t}-\mu$ and $\widetilde{\varepsilon}_{t}^{2}=\varepsilon_{t}^{2}$ - $\sigma_{\varepsilon}^{2}=\varepsilon_{\mathrm{t}}^{2}-1$. Then, we write the mean-adjusted form of GARCH process (2.1) in the ARMA form

$$
\tilde{h}_{t}=\sum_{i=1}^{n} f_{i} \tilde{h}_{t-i}+\sum_{i=1}^{n} g_{i} \xi_{t-i}
$$

where $\xi_{t}=\tilde{\varepsilon}_{t}^{2} h_{t}, f_{i}=\alpha_{i}+\beta_{i}$, and $g_{i}=\alpha_{i}$. 
A state-space form of an ARMA process comprises an observation equation and a state equation in terms of a state vector. Let $x_{t}=\left(x_{1, t}, \ldots, x_{n, t}\right)^{\top}$ denote an $\mathrm{n} \times 1$ state vector, where superscript $\mathrm{T}$ denotes transposition. Then, following Ansley and Kohn (1983), we can write ARMA equation (2.3) in statespace form, with observation equation $\tilde{h}_{t}=e_{1}^{\top} x_{t}$, where $e_{1}=(1, \odot, \ldots, \odot)^{\top}$ is the $\mathrm{n} \times 1$ vector with first element 1 and all other elements $\Theta$, and state equation

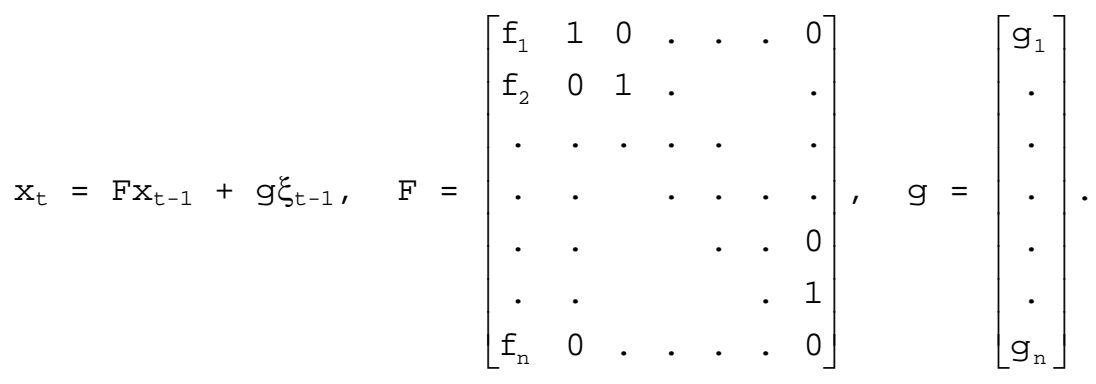

Observation equation $\tilde{h}_{t}=e_{1}^{\top} x_{t}$ implies $\tilde{h}_{t}$ is the first element of $x_{t}$. Because $F$ is a companion matrix, its eigenvalues are identical to the roots of the characteristic equation $\lambda^{n}-f_{1} \lambda^{n-1}-\ldots-f_{n-1} \lambda-f_{n}=0$ (Anderson, 1958, $p$. 177), so that stationarity condition $\sum_{i=1}^{n} f_{i}<1$ is equivalent to $\rho(F)<1$, where $\rho(F)$ denotes the spectral radius or maximal absolute eigenvalue of $F$. We say that the state equation (2.4) is stationary and the state-transition matrix $F$ is stable iff $\rho(F)<1$.

\section{4th-Moment Inequality and Eigenvalue Restrictions.}

We are interested in proving that derived restrictions for existence of $E y_{t}^{4}=E\left(\varepsilon_{t}^{4} h_{t}^{2}\right)$ are necessary and sufficient. However, we assume that $E \varepsilon_{t}^{4}$ exists; $E h_{t}^{2}$ and $E \widetilde{h}_{t}^{2}$ are linked by $E h_{t}^{2}=E \widetilde{h}_{t}^{2}+\mu^{2} ; E \widetilde{h}_{t}^{2}$ and $V$ are linked by $\mathrm{Eh}_{\mathrm{t}}^{2}=\mathrm{V}_{11}=\mathrm{e}_{1}^{\top} \mathrm{Ve}_{1}$; and, theorem 2 proves that $\mathrm{V}_{11}$ exists iff $\mathrm{V}$ exists, where $v_{11}$ and $V$ are defined two paragraphs below. Thus, proving that restrictions for existence of $\mathrm{Ey}_{\mathrm{t}}^{4}$ are necessary and sufficient reduces to proving such restrictions for $\mathrm{V}$.

We shall generally maintain two terminological conventions. First, when we write an inequality such as $" x \geq 0$ " we shall mean not only that the inequality holds but that the variable in it exists and, hence, is finite. 
Second, as noted before, we refer to assumptions (i) to (vii) on GARCH process (2.1) as "the GARCH assumptions."

Post-multiplying equation (2.4) by its transpose, taking unconditional expectation, $\mathrm{E}$, of the result, and apply independence assumption ( $\mathrm{Vi}$ ), so that $\mathrm{Ex}_{\mathrm{t}} \xi_{\mathrm{t}}^{\top}=\Theta_{\mathrm{n} \times 1}$, the $\mathrm{n} \times 1$ zero vector, implies the Lyapunov equation $\mathrm{V}=\mathrm{FVF}^{\top}$ $+\left(E \xi_{t}^{2}\right) g g^{\top}$, where $V=E x_{t} x_{t}^{\top}$. Then, $E \xi_{t}^{2}=E\left(\widetilde{\varepsilon}_{t}^{2}\right)^{2}\left(E \widetilde{h}_{t}^{2}+2 E \widetilde{h}_{t} \mu+\mu^{2}\right)=E\left(\widetilde{\varepsilon}_{t}^{2}\right)^{2}\left(E \widetilde{h}_{t}^{2}\right.$ $\left.+\mu^{2}\right)$, because $\tilde{\varepsilon}_{t}$ and $\tilde{h}_{t}$ are stochastically independent and $E \tilde{h}_{t}=\Theta$. In particular, $\tilde{\mathrm{h}}_{\mathrm{t}}^{2}$ depends only on constant parameters and variables dated before period $t$. Thus, the Lyapunov equation is equivalent to

$$
V=F V F^{\top}+\theta g e_{1}^{\top} V e_{1} g^{\top}+\mu^{2} \theta g g^{\top},
$$

where $\theta=E\left(\widetilde{\varepsilon}_{t}^{2}\right)^{2}=E \varepsilon_{t}^{4}-\left(E \varepsilon_{t}^{2}\right)^{2}=\sigma_{\varepsilon}^{4}-\left(\sigma_{\varepsilon}^{2}\right)^{2}=\sigma_{\varepsilon}^{4}-1>0$ (e.g., by Jensen's inequality). When $\varepsilon_{\mathrm{t}}$ is Gaussian, $\sigma_{\varepsilon}^{4}=3$, so that $\theta=2$.

Using $\operatorname{vec}(A B C)=\left[C^{\top} \otimes A\right] \operatorname{vec}(B)$, for matrices $A, B$, and $C$ conformable to the product ABC (Magnus and Neudecker, 1988, p. 30), where vec(.) denotes the column vectorization of a matrix (column 1 on top of column 2, etc.) and $\otimes$ denotes the Kronecker product. We state equation (3.1) equivalently as

$$
w=A W+\mu^{2} \theta(g \otimes g),
$$

where $\mathrm{W}=\operatorname{vec}(\mathrm{V})$ and $A=\mathrm{F} \otimes \mathrm{F}+\theta(\mathrm{g} \otimes \mathrm{g})\left(\mathrm{e}_{1} \otimes \mathrm{e}_{1}\right)^{\top}$.

Gantmacher (1959, pp. 50-57) discusses the following implications of irreducibility. A matrix or vector, $M$, is nonnegative $(M \geq 0)$ or positive ( $M>$ ๑) iff all of its elements are nonnegative or positive. A real, $n \times n$, and nonnegative matrix, $M$, is irreducible iff it has no invariant coordinate subspace with dimension less than $\mathrm{n}$. A theorem by Frobenius says that if $M$ is a real, $n \times n$, nonnegative, and irreducible matrix, then, $M$ has an eigenvalue, $\lambda$, and associated left or right eigenvector, $z$, such that $\lambda$ is real, positive, and equal to the maximal absolute eigenvalue of $M$ or $\lambda=\rho(M)$, and $z$ is real and positive. We need the following lemma.

LEMMA 1: Assume that GARCH assumptions (i) to (vii) hold. Then, matrices $F \geq 0$ and $A=F \otimes F+\theta(g \otimes g)\left(e_{1} \otimes e_{1}\right)^{\top} \geq \odot$ are irreducible. 
PROOF OF LEMMA 1: Let $e_{i}$ denote the $n \times 1$ elementary vector $i$, with one in position $i$ and zeroes elsewhere. The $n$-dimensional real vector space is spanned by $e_{1}, \ldots, e_{n}$. Consider $f_{1}=0, \ldots, f_{n-1}=0$ and $n$ successive mappings with $F$ starting from $e_{n}$ : $F_{n}=e_{n-1}, F^{2} e_{n}=e_{n-2}, \ldots, F^{n-1} e_{n}=e_{1}$, and $F^{n} e_{n}=f_{n} e_{n}$. The first n-1 mapped vectors are equal to the first $n-1$ elementary vectors, $e_{1}, \ldots, e_{n-1} ;$ because $f_{n}>\odot$, the last mapped vector lies in the space spanned by the last elementary vector, $e_{n}$. Thus, starting from $e_{n}$ and successively mapping $\mathrm{n}$ times with $\mathrm{F}$, the mapped vectors span the $\mathrm{n}$-dimensional real vector space. The same conclusion holds if we start the mappings from any other elementary vector and if $f_{1} \geq 0, \ldots, f_{n-1} \geq 0$. Thus, $F$ is irreducible. $F \otimes F \geq 0$ and, by a similar argument in $n \times n-b l o c k$ form, is irreducible. Because $F \otimes F \geq \odot$ and $\neq \odot, \quad \theta>\odot, \quad(g \otimes g)\left(e_{1} \otimes e_{1}\right)^{\top} \geq 0$ and $\neq \odot$, adding $(g \otimes g)\left(e_{1} \otimes e_{1}\right)^{\top} \geq \odot$ and $\neq \odot$ to $\mathrm{F} \otimes \mathrm{F}$ only increases the absolute values of the nonnegative elements of the first block-column of $F \otimes F$ and does not change its irreducibility. Thus, $A=$ $\mathrm{F} \otimes \mathrm{F}+\theta(\mathrm{g} \otimes \mathrm{g})\left(\mathrm{e}_{1} \otimes \mathrm{e}_{1}\right)^{\top}$ is irreducible and lemma 1 is proved.

The proof of lemma 1 depends on $f_{n}>0$, so that if $f_{n}=0$ because $\alpha_{n}=\beta_{n}$ $=\odot$, maintaining the lemma requires reducing $n$ until $f_{n}>\odot$.

\subsection{Necessary and Sufficient Inequality Restriction.}

Stationarity or $\rho(F)<1$ implies that $B=\sum_{i=0}^{\infty} F^{i} g g^{\top}\left(F^{\top}\right)^{i} \geq 0$ exists (Wilkinson, 1965, p. 59), so that equation (3.1) can be stated equivalently as

$$
\mathrm{V}=\left(\mathrm{v}_{11}+\mu^{2}\right) \theta \mathrm{B},
$$

where $v_{11}=e_{1}^{\top} V e_{1}$, the $(1,1)$ element of $V$. Pre-multiplying equation (3.3) by $\mathrm{e}_{1}^{\top}$, postmultiplying the result by $\mathrm{e}_{1}$, and rearranging, leads to equation (3.4), where $b_{11}=e_{1}^{\top} B_{1}$, the $(1,1)$ element of $B$. Thus, we have theorem 1 , which states the necessary and sufficient restriction $0<\theta b_{11}<1$ for existence of $E \tilde{h}_{t}^{2}=v_{11}>\odot$.

THEOREM 1: Assume that GARCH assumptions (i) to (vii) hold. Then, 


$$
v_{11}=\mu^{2}\left(\frac{\theta b_{11}}{1-\theta b_{11}}\right)>0
$$

exists iff, in addition, $\odot<\theta b_{11}<1$.

PROOF OF THEOREM 1: The GARCH assumptions imply that $\mu>0$ and $\theta>0$ exist. In particular, stationarity implies that $B \geq 0$ exists. Because $F \geq 0$ and is irreducible (lemma 1 ) and $g \geq 0$ and $\neq 0, b_{11}=e_{1}^{\top} B_{1} \geq e_{1}^{\top} F^{j} g g^{\top}\left(F^{\top}\right)^{j} e_{1}>\odot$, for some positive integer $j$. Thus, $\mu^{2} \theta b_{11}>0$ and equation (3.4) implies that $v_{11}>$ $\odot$ exists iff, in addition, $\theta b_{11}<1$ and theorem 1 is proved.

Next, equation (3.3) implies theorem 2, which links the existence of $V$ with the existence of $v_{11}$.

THEOREM 2: Assume that GARCH assumptions (i) to ( $\mathrm{vi}$ ) hold. Then, $\mathrm{V} \geq 0$ exists iff $v_{11}>0$ exists.

PROOF OF THEOREM 2: Suppose that $\mathrm{V} \geq 0$ exists. Then, $\mathrm{v}_{11}=\mathrm{e}_{1}^{\top} \mathrm{Ve}_{1} \geq \odot$ exists. The GARCH assumptions imply that $\mu>0$ and $\theta>0$ exist, that $F$ is stable, nonnegative, and irreducible, and that $g \geq 0$ and $\neq 0$. These properties imply that $B \geq 0$ and $b_{11}>0$ exist. Thus, equation (3.3) implies that $v_{11}=\left(v_{11}+\right.$ $\left.\mu^{2}\right) \theta b_{11} \geq \mu^{2} \theta b_{11}>\odot$ and necessity is proved. Suppose that $v_{11}>\odot$ exists. The GARCH assumptions imply that $\mu>\odot, \theta>0$, and $B \geq 0$ exist. Thus, equation (3.3) implies that $V \geq 0$ exists and sufficiency and theorem 2 are proved.

\subsection{Necessary and Sufficient Eigenvalue Restriction.}

Equation (3.2) has the unique solution $w=\mu^{2} \theta\left(I_{n^{2}}-A\right)^{-1}(g \otimes g)$ iff $I_{n^{2}}-A$ is nonsingular, where $I_{n^{2}}$ denotes the $n^{2} \times n^{2}$ identity matrix, and this occurs iff $A$ has no eigenvalue equal to one, because each eigenvalue of $I_{n^{2}}-A$ is one minus an eigenvalue of $A$. Theorem 3 tells us that $v_{11}>\odot$ only if $\rho(A)<1$, because otherwise $w$ might have unacceptable negative values. 
THEOREM 3: Assume that GARCH assumptions (i) to ( $\mathrm{vii}$ ) hold. Then, $w=\operatorname{vec}(\mathrm{V})$ $\geq \odot$ and $v_{11}=w_{1}>\odot$ exist, where $w_{1}$ denotes the first element of $w$, iff, in addition, $\rho(A)<1$.

PROOF OF THEOREM 3: Suppose that $\rho(A)<1$. Then, $\rho\left(I_{n^{2}}-A\right)<1$, so that $w=$ $\mu^{2} \theta\left(I_{n^{2}}-A\right)^{-1}(g \otimes g)$ uniquely solves equation (3.2). The GARCH assumptions imply that $\theta>\odot$ and $\mu>0$ exist. The solution $w=\mu^{2} \theta\left(I_{n^{2}}-A\right)^{-1}(g \otimes g)$ implies that $w=$ $\mu^{2} \theta\left(I_{n^{2}}+A+A^{2}+\ldots\right)(g \otimes g)$. The irreducibility of $A$ implies that $\left(e_{1} \otimes e_{1}\right)^{\top} A^{j}(g \otimes g)>0$, for some positive integer $j$. See the proof of lemma 1. Thus, $v_{11}=W_{1}>0$ and sufficiency is proved. Suppose that $\delta \mathbf{X}^{\top}=\mathrm{X}^{\top} \mathrm{A}$, where $\delta$ is a maximal eigenvalue of $A$, so that $|\delta|=\rho(A)$, and $x$ is an associated left eigenvector of $A$. Because $A$ is real, nonnegative, and irreducible, Frobenius's theorem (Gantmacher, 1959, pp. 50-57) implies that $\delta$ and $x$ are real and positive. Pre-multiplying equation (3.2) by $x^{\top}$ implies that

$$
(1-\delta) x^{\top} w=\mu^{2} \theta x^{\top}(g \otimes g) \text {. }
$$

However, $\mathrm{x}>\Theta, \mathrm{w} \geq \Theta, \mathrm{w}_{1}>\Theta$, and $\mathrm{g} \otimes \mathrm{g} \geq 0$ and $\neq \Theta$ imply that $\mathrm{x}^{\top} \mathrm{w}>\Theta$ and $X^{\top}(g \otimes g)>\odot$. Thus, $\mu^{2} \theta>\odot$ implies that $1-\delta=\mu^{2} \theta x^{\top}(g \otimes g) / X^{\top} W>\odot$, so that equation (3.5) implies that $\delta=\rho(A)<1$ and necessity and theorem 3 are proved.

Putting together theorems 1 to 3 implies that $\theta b_{11}<1$ and $\rho(A)<1$ are equivalent necessary and sufficient restrictions for existence of the 4 th moment of the underlying variable of GARCH process (2.1). We state this conclusion formally as corollary 1.

COROLLARY 1: Assume that GARCH assumptions (i) to (viii) hold. Then, $\theta \mathrm{b}_{11}<1$ iff $\rho(A)<1$.

Statistics tells us that a 4th moment exists only if the corresponding 2nd moment exists. Here, this means that $\rho(A)<1$ only if $\rho(F)<1$. Theorem 4 generalizes this result and shows that 4th-moment existence implies stronger restrictions than stationarity or 2nd-moment existence. For example, under 
the GARCH assumptions, stationarity occurs iff $B \geq 0$ and $b_{11}>\odot$ exist, but 4th-moment existence occurs iff, in addition, $\theta b_{11}<1$.

THEOREM 4: $\rho(F)^{2}<\rho(A)$, where $A=F \otimes F+\theta(g \otimes g)\left(e_{1} \otimes e_{1}\right)^{\top}$.

PROOF of THEOREM 4: Let $\lambda^{2}(z \otimes z)^{\top}=(z \otimes z)^{\top}(F \otimes F)$ and $\delta x=A x$, where $\lambda$ and $\delta$ are maximal eigenvalues of $F$ and $A$, so that $|\lambda|=\rho(F)$ and $|\delta|=\rho(A)$, and $z$ and $x$ are associated left and right eigenvalues of $F$ and $A$. The GARCH assumptions and Frobenius's theorem imply that $\lambda, \delta, z$, and $\mathrm{x}$ are real and positive. Premultiplying $\delta x=A x$ by $(z \otimes z)^{\top}$ and rearranging implies that

$$
\left(\delta-\lambda^{2}\right)(z \otimes z)^{\top} x=\theta\left(z^{\top} g\right)^{2} x_{1}
$$

where $x_{1}$ denotes the first element of $x$. However, $\theta>0, z>0, x>0, g \geq 0$, and $g \neq \odot$, imply that $(z \otimes z)^{\top} x>\odot, z^{\top} g>\odot$, and $x_{1}>0$, so that equation (3.6) implies that $\delta-\lambda^{2}=\theta\left(z^{\top} g\right)^{2} x_{1} /(z \otimes z)^{\top} x>0$ and theorem 4 is proved.

\section{Illustration of the 4th-Moment Inequality Restriction.}

We now illustrate equation (3.4) in table 1 with six $\operatorname{GARCH}(1,1)$ processes from the literature. To do this, we first write $b_{11}$ in terms of $a$ finite number of basic arithmetic operations on $f_{i}$ and $g_{i}$. Pre-multiplying $B=$ $\sum_{i=0}^{\infty} F^{i} g g^{\top}\left(F^{\top}\right)^{i}$ by $F$, post-multiplying the result by $F^{\top}$, subtracting the result from the initial equation for $B$, and rearranging, implies that

$$
B=F B F^{\top}+g g^{\top},
$$

which is a Lyapunov equation, linear in B.

The companion form of $F$ suggests that equation (3.7) can be solved for $b_{11}$ by eliminating elements of $B$ from "back to front" until only $b_{11}$ remains. Although such an approach might generally be overly complex and impractical, it is easily applied when $n=2$. We consider $n=2$ in part because it includes nearly all the GARCH processes we have seen in the empirical literature. For $n=2$, equation (3.7) implies that 


$$
b_{11}=\frac{\left(1-f_{2}\right)\left(g_{1}^{2}+g_{2}^{2}\right)+2 f_{1} g_{1} g_{2}}{\left(1-f_{2}\right)\left(1-f_{1}^{2}-f_{2}^{2}\right)-2 f_{1}^{2} f_{2}}
$$

The GARCH nonegativity assumptions $f_{i} \geq \odot$ and $g_{i} \geq \odot$ and the stationarity assumption $\sum_{i=1}^{n} f_{i}<1$ imply that the numerator and denominator in equation (3.8) are both positive.

Although a GARCH process linearly transforms squared disturbances, $\varepsilon_{\mathrm{t}}^{2}$, into squared variables, $y_{t}^{2}$, it nonlinearly transforms $\varepsilon_{t}$ to $y_{t}$, so that $y_{t}$ is non-Gaussian even when $\varepsilon_{t}$ is Gaussian. We may consider this nonpreservation of Gaussianity in terms of kurtosis, by considering equation (3.4) as

$$
\frac{\mathrm{k}_{\mathrm{y}}}{\mathrm{k}_{\varepsilon}}=\frac{\theta \mathrm{b}_{11}}{1-\theta \mathrm{b}_{11}}+1
$$

where $\mathrm{k}_{\mathrm{y}}=\mathrm{Ey}_{\mathrm{t}}^{4} /\left(\mathrm{Ey}_{\mathrm{t}}^{2}\right)^{2}$ and $\mathrm{k}_{\varepsilon}=E \varepsilon_{\mathrm{t}}^{4} /\left(E \varepsilon_{\mathrm{t}}^{2}\right)^{2}$ are the kurtoses of $\mathrm{y}_{\mathrm{t}}$ and $\varepsilon_{\mathrm{t}}$. Equation (3.9) implies that a GARCH transformation always increases kurtosis.

Table 1 considers only the case of $n=1$ or $\operatorname{GARCH}(1,1)$ processes, which covers most of the empirical literature. Some exceptions are Engle (1982) and Geweke (1988) who consider nonstationary $\operatorname{GARCH}(\odot, 4)$ and $\operatorname{GARCH}(\odot, 2)$ processes which we exclude because they do not have 4th moments. In table 1, we assume that $\varepsilon_{t} \sim N(\odot, 1)$ (Gaussian, zero mean, unit variance) even though Bollerslev, Drost and Klaassen, and Hsieh assume that $\varepsilon_{t}$ is non-Gaussian. The cases in table 1 are ordered by increasing $f_{1}=\alpha_{1}+\beta_{1}$ and, for $n=1, \sigma_{\varepsilon}^{2}=1$, and $\theta=$ 2, equations (3.8) and (3.9) reduce to

$$
\mathrm{b}_{11}=\frac{\mathrm{g}_{1}^{2}}{1-\mathrm{f}_{1}^{2}}=\frac{\alpha_{1}^{2}}{1-\left(\alpha_{1}+\beta_{1}\right)^{2}}
$$

$$
\frac{\mathrm{k}_{\mathrm{y}}}{\mathrm{k}_{\varepsilon}}=\frac{1-\mathrm{f}_{1}^{2}}{1-\mathrm{f}_{1}^{2}-2 \mathrm{~g}_{1}^{2}}=\frac{1-\left(\alpha_{1}+\beta_{1}\right)^{2}}{1-\left(\alpha_{1}+\beta_{1}\right)^{2}-2 \alpha_{1}^{2}} .
$$

Table 1 contains values of $\theta b_{11}$ and $k_{y} / k_{\varepsilon}$, for $\theta=2$, according to equations (3.10) and (3.11) for $\operatorname{six} \operatorname{GARCH}(1,1)$ processes from the literature. 
Table 1: Examples of $\theta b_{11}$ and $k_{y} / k_{\varepsilon}$ for $\operatorname{GARCH}(1,1)$ processes and $\theta=2$.

\begin{tabular}{|c|l|c|c|c|c|c|}
\hline Case & \multicolumn{1}{|c|}{ Date, Authors } & $\alpha_{1}$ & $\beta_{1}$ & $\mathrm{f}_{1}$ & $\theta \mathrm{b}_{11}$ & $\mathrm{k}_{\mathrm{y}} / \mathrm{k}_{\varepsilon}$ \\
\hline 1 & 1982, Engle & .955 & .000 & .955 & 20.6 & $\infty$ \\
\hline 2 & 1986, Bollerslev & .135 & .829 & .964 & .516 & 2.07 \\
\hline 3 & 1989, Baillie-Bollerslev & .061 & .910 & .971 & .130 & 1.15 \\
\hline 4 & 1987, Bollerslev & .057 & .921 & .978 & .149 & 1.18 \\
\hline 5 & 1997, Drost-Klaassen & .052 & .932 & .984 & .170 & 1.21 \\
\hline 6 & 1989, Hsieh & .191 & .806 & .997 & 12.2 & $\infty$ \\
\hline
\end{tabular}

Table 1 depicts small $\alpha_{1}$ (cases 2 to 5 ) associated with small $\theta b_{11}<1$, for $\theta=2$, and finite $k_{y} / k_{\varepsilon}$, larger and large $\alpha_{1}$ (cases 6 and 1 ) associated with large $\theta b_{11}>1$ and infinite $k_{y} / k_{\varepsilon}$, and, this pattern appears to be independent of the value of $\beta_{1}$ and to depend more on the value of $\alpha_{1}$ (compare cases 1 and 6 with cases 2 to 5 ). The pattern is confirmed in figure 1 , which depicts the feasible area of $\alpha_{1}$ and $\beta_{1}$ in which the underlying variable in a scalar $\operatorname{GARCH}(1,1)$ process with $\varepsilon_{t} \sim N(\odot, 1)$ has 2 nd and 4 th moments. The feasible area for stationarity or 2nd-moment existence is between the horizontal- $\alpha_{1}$ axis, the vertical- $\beta_{1}$ axis, and the straight line $\beta_{1}=1-\alpha_{1}$; the feasible subarea for 4 th-moment existence is between the axes and the curved line $\beta_{1}=-\alpha_{1}+\sqrt{1-2 \alpha_{1}^{2}}$. Evidently, 4th-moment existence restricts both $\alpha_{1}$ and $\beta_{1}$, but restricts $\alpha_{1}$ more: if a univariate $\operatorname{GARCH}(1,1)$ process is stationary and its underlying variable has a 4 th moment, then, a minimum $\alpha_{1}=$ $\odot$ implies a maximum $\beta_{1}=1$, but a minimum $\beta_{1}=\odot$ implies a maximum $\alpha_{1}=1 / \sqrt{3} \cong$ .5773, because $\beta_{1}$ is either real and negative or complex with a negative real part when $1 / \sqrt{3}<\alpha_{1}<1$. Adding 4 th-moment existence to 2nd-moment existence, reduces the feasible area of $\alpha_{1}$ and $\beta_{1}$ by about one third. Adding more GARCH lags $(n>1)$ or higher even-moment existence further reduces the feasible area of $\alpha_{1}$ and $\beta_{1}$. 


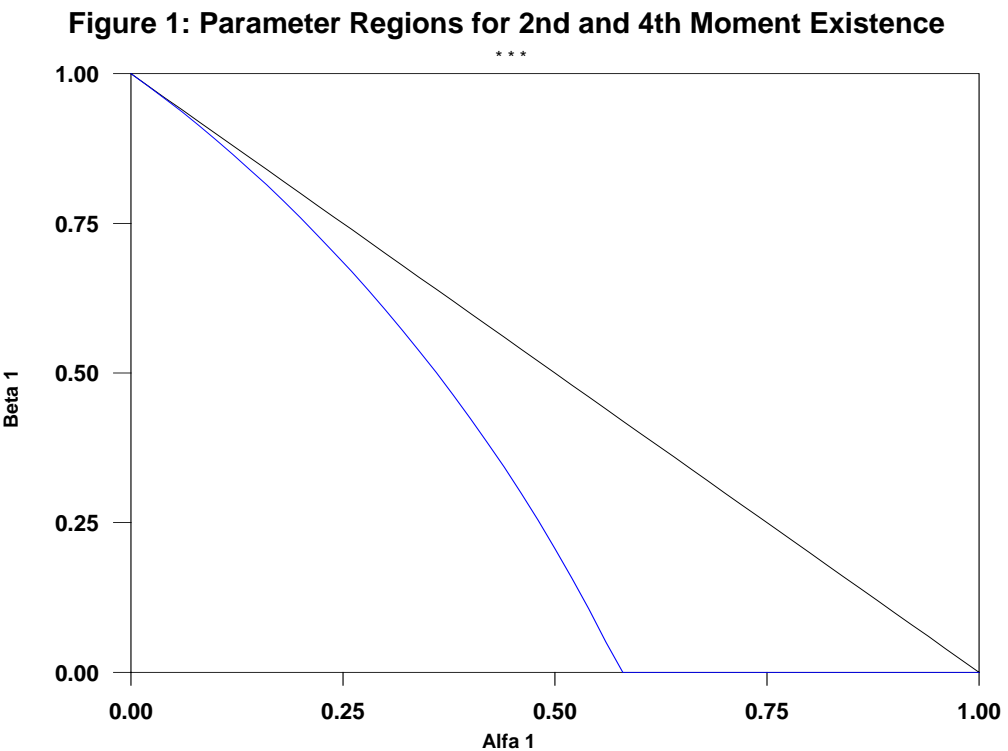

Equations (3.8) to (3.11), table 1, and figure 1, illustrate an important advantage of inequality restriction $\theta b_{11}<1$ over eigenvalue restriction $\rho(A)<1$. For $n=2$, equation (3.8) states $\theta b_{11}<1$ in terms of $a$ finite number of basic arithmetic operations on the GARCH parameters, and, for $n=1$, table 1 evaluates and figure 1 depicts this inequality. In principle, this could be done for any $n$, but, when $n$ is large enough, algebraic detail becomes overwhelming. By contrast, generally, an eigenvalue restriction can be written out explicitly in terms of the GARCH parameters only when $\mathrm{n} \leq 3$. When $\mathrm{n}>3$, this can be done only in unlikely cases in which the characteristic polynomial of $A=F \otimes F+\theta(g \otimes g)\left(e_{1} \otimes e_{1}\right)^{\top}$ factors into polynomials of degree $\leq 3$.

\section{Conclusion.}

Either equation (3.4) or (3.9) indicate that the underlying variable, $\mathrm{y}_{\mathrm{t}}$, of univariate GARCH process (2.1) has a 4th moment iff $\theta<\theta \mathrm{b}_{11}<1$ exists. Non-Gaussian disturbances without 4th moments, in particular, stable Paretian disturbances, have been considered (McCulloch, 1997; Rachev and Mittnik, 2000 ). Whether or not a disturbance has a 4th moment, in practice we would like to know whether an estimated GARCH process preserves existence of a disturbance's 4 th moment. This would involve developing a statistical test of 
$\theta b_{11}<1$ which accounts for the sampling variability of an estimated $\theta b_{11}$, based on the estimated GARCH parameters. Ideally, this would be an easily formed test, based on standard parameter estimates, along the lines of Dickey and Fuller's (1979) test of unit-root nonstationarity of an autoregressive linear time-series process.

Various papers in the literature claim to prove necessity and sufficiency of restrictions for 4th-moment existence in univariate GARCH processes, but most do not discuss any sort of nonnegativity (e.g., Hafner, 2003). Here, we see the crucial role of nonnegativity in the necessity proofs. Nonnegativity also figures crucially in existence proofs of stationarity of GARCH processes, but there nonnegativity occurs more simply, as is seen in equation (2.2). Bougerol and Picard (1992) and Ling and McAleer (2002) are exceptions in the literature for discussing nonnegativity in their respective existence proofs for 2nd and 4th moments of GARCH processes.

The state-space form of a GARCH process used here is simpler than the forms used by Bougerol and Picard (1992), Ling and McAleer (2002), and Mittnik, Paolella, and Rachev (2002), because the transition matrix $F$ is nonstochastic. Thus, the proofs here are based on more elementary concepts and are simpler. For example, there is no need to consider a stochastic Lyapunov exponent and to verify its convergence, as in Bougerol and Picard (1992).

Finally, for any positive integer $\mathrm{m}$, it would be interesting to generalize present restrictions to necessary and sufficient restrictions for existence of unique 2mth-moments of the underlying variable of a univariate GARCH process (cf., Ling and McAleer, 2002).

\section{REFERENCES}

Anderson, T.W. (1958), The Statistical Analysis of Time Series, New York, NY: John Wiley \& Sons.

Ansley, C.F. and R. Kohn (1983), "Exact Likelihood of Vector Autoregressive Moving-Average Process with Missing or Aggregated Data," Biometrika 70: 275278.

Baillie, R. and T. Bollerslev (1989), "The Message in Daily Exchange Rates: A Conditional Variance Tale," Journal of Business and Economic statistics 7: 297-305.

Baillie, R. and T. Bollerslev (1992), "Prediction in Dynmaic Models with Time-Dependent Conditional Variances," Journal of Econometrics 52: 91-113. 
Bollerslev, T, (1986), "Generalized Autoregressive Conditional Heteroskedasticity," Journal of Econometrics 31: 307-327.

Bollerslev, T. (1987), "A Conditionally Heteroskedastic Time Series Model for Speculative Prices and Rates of Return," Review of Economics and Statistics 69: $542-547$.

Bougerol, P. and N. Picard (1992), "Stationarity of GARCH Processes and of Some Nonnegative Time Series," Journal of Econometrics 52: 115-127.

Dickey, D.A. and W.A. Fuller (1979), "Distribution of the Estimates for Autoregressive Time Series with a Unit Root," Journal of the American Statistical Association 74: 427-431.

Drost, F.C. and C.A.J. Klaassen (1997), "Efficient Estimation in Semiparametric GARCH Models," Journal of Econometrics 81: 193-221.

Engle, R.F. (1982), "Autoregressive Conditional Heteroskedasticity with Estimates of the Variance of United Kingdom Inflation," Econometrica 50: 9871008 .

Gantmacher, F.R. (1959), The Theory of Matrices, Vol. 2, Providence, RI: Chelsea Publishing.

Geweke, J. (1988), "Exact Inference in Models with Autoregressive Conditional Heteroskedasticity," pp. 73-104 in W.A. Barnett, E.R. Berndt, and H. White (eds.), Dynamic Econometric Modelling, Cambridge, United Kingdom: Cambridge University Press.

Hafner, C. (2003), "Fourth Moment Structure of Multivariate GARCH Models," Journal of Financial Econometrics 1: 26-54.

He, C. and T. Terasvirta (1999), "Fourth Moment Structure of the GARCH $(p, q)$ Process," Econometric Theory 15: 824-846.

Hsieh, D. (1989), "Modelling Heteroskedasticity in Daily Foreign-Exchange Rates," Journal of Business and Economic Statistics 7: 307-317.

Karanasos, M. (1999), "The Second Moment and the Autocovariance Function of the Squared Errors of the GARCH Model," Journal of Econometrics 90: 63-76.

Ling, S. (1999), "On the Probabilistic Properties of a Double Threshold ARMA Conditional Heteroskedasticity Model," Journal of Applied Probability 36: $688-705$.

Ling, S. and M. McAleer (2002), "Necessary and Sufficient Moment Conditions for the $\operatorname{GARCH}(r, s)$ and Asymmetric Power $\operatorname{GARCH}(r, s)$ Models," Econometric Theory 18: 722-729.

Magnus, J.R. and H. Neudecker (1988), Matrix Differential Calculus with Applications in Statistics and Econometrics, New York, NY: John Wiley \& Sons.

Mandelbrot, B. (1963a), "New Methods in Statistical Economics," Journal of Political Economy 71: 421-440.

Mandelbrot, B. (1963b), "The Variation of Certain Speculative Prices," Journal of Business 36: 394-419. 
McCulloch, J.H. (1997), "Measuring Tail Thickness in order to Estimate the Stable Index $\alpha$ : A Critique," Journal of Business and Economic Statistics 15: 74-81.

Milhoj, A. (1985), "The Moment Structure of ARCH Processes," Scandinavian Journal of Statistics 12: 281-292.

Mittnik, S., M.S. Paolella, and S. Rachev (2002), "Stationarity of Stable Power-GARCH Processes," Journal of Econometrics 106: 97-107.

Nelson, D.B. (1990), "Stationarity and Persistence in the $\operatorname{GARCH}(1,1)$ Model," Econometric Theory 6: 318-334.

Rachev, S. and S. Mittnik (2000), Stable Paretian Models in Finance, New York, NY: John Wiley \& Sons.

Wilkinson, J.H. (1965), The Algebraic Eigenvalue Problem, oxford, U.K.: Clarendon Press. 


\section{CESifo Working Paper Series}

(for full list see www.cesifo-group.de)

1437 Martin Kolmar and Volker Meier, Intra-Generational Externalities and InterGenerational Transfers, March 2005

1438 M. Hashem Pesaran and Takashi Yamagata, Testing Slope Homogeneity in Large Panels, March 2005

1439 Gjermund Nese and Odd Rune Straume, Industry Concentration and Strategic Trade Policy in Successive Oligopoly, April 2005

1440 Tomer Blumkin and Efraim Sadka, A Case for Taxing Education, April 2005

1441 John Whalley, Globalization and Values, April 2005

1442 Denise L. Mauzerall, Babar Sultan, Namsoug Kim and David F. Bradford, Charging $\mathrm{NO}_{x}$ Emitters for Health Damages: An Exploratory Analysis, April 2005

1443 Britta Hamburg, Mathias Hoffmann and Joachim Keller, Consumption, Wealth and Business Cycles in Germany, April 2005

1444 Kohei Daido and Hideshi Itoh, The Pygmalion Effect: An Agency Model with Reference Dependent Preferences, April 2005

1445 John Whalley, Rationality, Irrationality and Economic Cognition, April 2005

1446 Henning Bohn, The Sustainability of Fiscal Policy in the United States, April 2005

1447 Torben M. Andersen, Is there a Role for an Active Fiscal Stabilization Policy? April 2005

1448 Hans Gersbach and Hans Haller, Bargaining Power and Equilibrium Consumption, April 2005

1449 Jerome L. Stein, The Transition Economies: A NATREX Evaluation of Research, April 2005

1450 Raymond Riezman, John Whalley and Shunming Zhang, Metrics Capturing the Degree to which Individual Economies are Globalized, April 2005

1451 Romain Ranciere, Aaron Tornell and Frank Westermann, Systemic Crises and Growth, April 2005

1452 Plutarchos Sakellaris and Focco W. Vijselaar, Capital Quality Improvement and the Sources of Growth in the Euro Area, April 2005

1453 Kevin Milligan and Michael Smart, Regional Grants as Pork Barrel Politics, April 2005 
1454 Panu Poutvaara and Andreas Wagener, To Draft or not to Draft? Efficiency, Generational Incidence, and Political Economy of Military Conscription, April 2005

1455 Maurice Kugler and Hillel Rapoport, Skilled Emigration, Business Networks and Foreign Direct Investment, April 2005

1456 Yin-Wong Cheung and Eiji Fujii, Cross-Country Relative Price Volatility: Effects of Market Structure, April 2005

1457 Margarita Katsimi and Thomas Moutos, Inequality and Relative Reliance on Tariffs: Theory and Evidence, April 2005

1458 Monika Bütler, Olivia Huguenin and Federica Teppa, Why Forcing People to Save for Retirement may Backfire, April 2005

1459 Jos Jansen, The Effects of Disclosure Regulation of an Innovative Firm, April 2005

1460 Helge Bennmarker, Kenneth Carling and Bertil Holmlund, Do Benefit Hikes Damage Job Finding? Evidence from Swedish Unemployment Insurance Reforms, May 2005

1461 Steffen Huck, Kai A. Konrad and Wieland Müller, Merger without Cost Advantages, May 2005

1462 Louis Eeckhoudt and Harris Schlesinger, Putting Risk in its Proper Place, May 2005

1463 Hui Huang, John Whalley and Shunming Zhang, Trade Liberalization in a Joint Spatial Inter-Temporal Trade Model, May 2005

1464 Mikael Priks, Optimal Rent Extraction in Pre-Industrial England and France - Default Risk and Monitoring Costs, May 2005

1465 François Ortalo-Magné and Sven Rady, Heterogeneity within Communities: A Stochastic Model with Tenure Choice, May 2005

1466 Jukka Pirttilä and Sanna Tenhunen, Pawns and Queens Revisited: Public Provision of Private Goods when Individuals make Mistakes, May 2005

1467 Ernst Fehr, Susanne Kremhelmer and Klaus M. Schmidt, Fairness and the Optimal Allocation of Ownership Rights, May 2005

1468 Bruno S. Frey, Knight Fever - Towards an Economics of Awards, May 2005

1469 Torberg Falch and Marte Rønning, The Influence of Student Achievement on Teacher Turnover, May 2005

1470 John Komlos and Peter Salamon, The Poverty of Growth with Interdependent Utility Functions, May 2005 
1471 Hui Huang, Yi Wang, Yiming Wang, John Whalley and Shunming Zhang, A Trade Model with an Optimal Exchange Rate Motivated by Current Discussion of a Chinese Renminbi Float, May 2005

1472 Helge Holden, Lars Holden and Steinar Holden, Contract Adjustment under Uncertainty, May 2005

1473 Kai A. Konrad, Silent Interests and All-Pay Auctions, May 2005

1474 Ingo Vogelsang, Electricity Transmission Pricing and Performance-Based Regulation, May 2005

1475 Spiros Bougheas and Raymond Riezman, Trade and the Distribution of Human Capital, June 2005

1476 Vesa Kanniainen, Seppo Kari and Jouko Ylä-Liedenpohja, The Start-Up and Growth Stages in Enterprise Formation: The "New View" of Dividend Taxation Reconsidered, June 2005

1477 M. Hashem Pesaran, L. Vanessa Smith and Ron P. Smith, What if the UK had Joined the Euro in 1999? An Empirical Evaluation Using a Global VAR, June 2005

1478 Chang Woon Nam and Doina Maria Radulescu, Effects of Corporate Tax Reforms on SMEs' Investment Decisions under the Particular Consideration of Inflation, June 2005

1479 Panos Hatzipanayotou, Sajal Lahiri and Michael S. Michael, Globalization, CrossBorder Pollution and Welfare, June 2005

1480 John Whalley, Pitfalls in the Use of Ad valorem Equivalent Representations of the Trade Impacts of Domestic Policies, June 2005

1481 Edward B. Barbier and Michael Rauscher, Trade and Development in a Labor Surplus Economy, June 2005

1482 Harrie A. A. Verbon and Cees A. Withagen, Tradable Emission Permits in a Federal System, June 2005

1483 Hendrik Hakenes and Andreas Irmen, On the Long-Run Evolution of Technological Knowledge, June 2005

1484 Nicolas Schmitt and Antoine Soubeyran, A Simple Model of Brain Circulation, June 2005

1485 Carsten Hefeker, Uncertainty, Wage Setting and Decision Making in a Monetary Union, June 2005

1486 Ondřej Schneider and Jan Zápal, Fiscal Policy in New EU Member States - Go East, Prudent Man!, June 2005

1487 Christian Schultz, Virtual Capacity and Competition, June 2005 
1488 Yvan Lengwiler and Elmar Wolfstetter, Bid Rigging - An Analysis of Corruption in Auctions, June 2005

1489 Johannes Becker and Clemens Fuest, Does Germany Collect Revenue from Taxing Capital Income?, June 2005

1490 Axel Dreher and Panu Poutvaara, Student Flows and Migration: An Empirical Analysis, June 2005

1491 Bernd Huber and Marco Runkel, Interregional Redistribution and Budget Institutions under Asymmetric Information, June 2005

1492 Guido Tabellini, Culture and Institutions: Economic Development in the Regions of Europe, July 2005

1493 Kurt R. Brekke and Michael Kuhn, Direct to Consumer Advertising in Pharmaceutical Markets, July 2005

1494 Martín Gonzalez-Eiras and Dirk Niepelt, Sustaining Social Security, July 2005

1495 Alfons J. Weichenrieder, (Why) Do we need Corporate Taxation?, July 2005

1496 Paolo M. Panteghini, S-Based Taxation under Default Risk, July 2005

1497 Panos Hatzipanayotou and Michael S. Michael, Migration, Tied Foreign Aid and the Welfare State, July 2005

1498 Agata Antkiewicz and John Whalley, BRICSAM and the Non-WTO, July 2005

1499 Petr Hedbávný, Ondřej Schneider and Jan Zápal, A Fiscal Rule that has Teeth: A Suggestion for a 'Fiscal Sustainability Council' underpinned by the Financial Markets, July 2005

1500 J. Atsu Amegashie and Marco Runkel, Sabotaging Potential Rivals, July 2005

1501 Heikki Oksanen, Actuarial Neutrality across Generations Applied to Public Pensions under Population Ageing: Effects on Government Finances and National Saving, July 2005

1502 Xenia Matschke, Costly Revenue-Raising and the Case for Favoring Import-Competing Industries, July 2005

1503 Horst Raff and Nicolas Schmitt, Why Parallel Trade may Raise Producers Profits, July 2005

1504 Alberto Bisin and Piero Gottardi, Efficient Competitive Equilibria with Adverse Selection, July 2005

1505 Peter A. Zadrozny, Necessary and Sufficient Restrictions for Existence of a Unique Fourth Moment of a Univariate GARCH(p,q) Process, July 2005 